Círculo de Lingüística Aplicada a la Comunicación ISSN: 1576-4737

Prague 2018: 16th International Workshop on Treebanks and Linguistic Theories TLT

https://ufal.mff.cuni.cz/tlt16/

TLT serves as a venue for new and ongoing research on the topic of linguistics and treebanks. The 16th edition of TLT will return to the heart of Europe, at Charles University, Prague, in January 2018.

For 16 years now, TLT has served as a venue for new and ongoing high-quality work related to syntactically-annotated corpora, i.e., treebanks; with a focus on all the aspects of treebanking - descriptive, theoretical, formal and computational but also going beyond treebanks, including other levels of annotation such as frame semantics, coreference or events, to name only a few.

\title{
Important dates
}

23 June 2017: Submission system open

9 November 2017: Deadline for submissions, registration open

21 November 2017: Notification of acceptance

11 December 2017: Final papers due

23-24 January 2018: The conference

\section{Local chair}

Jan Hajic

\section{Vienna 2018: CRH ("Corpus-based Research in the Humanities")}

\section{Co-located workshop: CRH in Vienna}

Since TLT in Warsaw, the CRH ("Corpus-based Research in the Humanities") workshop has been collocated with TLT. This year, it takes place just a couple hours away - in Vienna, Austria, immediately following the TLT.

https://www.oeaw.ac.at/ac/crh2/ 


\section{Conference Overview}

The second edition of the workshop on "Corpus-based Research in the Humanities" (CRH) will be held in Vienna (Austria) on January 25th-26th 2018 and will be hosted by Academy Corpora of the Austrian Academy of Sciences.

After the first edition in Warsaw (Poland) in 2015 the CRH workshop continues the series of workshops previously named "Annotation of Corpora for Research in the Humanities" (ACRH). Three editions of ACRH were held, respectively in 2011 (Heidelberg, Germany), 2012 (Lisbon, Portugal) and 2013 (Sofia, Bulgaria). The first edition of $\mathrm{CRH}$ was held in Warsaw (Poland) in 2015. All the previous editions were co-located with the internaitonal workshop on Treebanks and Linguistic Theories (TLT).

This year, the 16th edition of TLT (https://ufal.mff.cuni.cz/tlt16/) takes place just a couple hours away from CRH - in Prague (Czech Republic) - and immediately before CRH (on 23-24 January 2018). We hope that the two events will have several participants in common.

CRH wants to be a meeting place for both scholars from Computational Linguistics and from the Humanities (especially, Digital Humanities). Although the two research areas share a number of common topics, there is still limited collaboration between the two communities. Since the empirical evidence provided by corpora plays a central role in both disciplines, we believe that a workshop focussed on the different uses of (different kinds of) corpus data in the Humanities might represent a valid opportunity to make the two communities meet, discuss and compare their interests, methods and aims.

This year, CRH will have a special topic concerning time and space annotation in textual data.

\section{Invited speakers}

Tara L. Andrews, University of Wien, Austria

(http://www.univie.ac.at/Geschichte/htdocs2/site/arti.php/91079)

James Pustejovsky, Brandeis University, MA, USA (http://jamespusto.com/) 